\title{
Necrotizing 'sarcoidal' angiitis and granulomatosis of the lung
}

\author{
J. G . S T E P H E N, M . V. B R A I M B R I D G E, \\ B. COR R I N,S.P. W I L K I N S N, D. D A Y, a n d \\ W. F. W H I M S T E R \\ St. Thomas' Hospital and King's College Hospital, London
}

\begin{abstract}
Stephen, J. G., Braimbridge, M. V., Corrin, B., Wilkinson, S. P., Day, D., and Whimster W. F. (1976). Thorax, 31, 356-360. Necrotizing 'sarcoidal' angiitis and granulomatosis of the lung. A case of necrotizing sarcoid angiitis and granulomatosis (NSG) presenting as a peripheral lung 'tumour' in a 63-year-old man is recorded, and the clinical and pathological features are compared with those of Liebow's original cases. Resection may be avoided if the diagnosis is made by biopsy as the disease is possibly steroid responsive and the prognosis appears favourable. The aetiology is obscure but an immune disturbance is suspected.
\end{abstract}

Liebow (1973) has recently defined five forms of pulmonary angiitis and granulomatosis not produced by known infec'ious agents or associated with rheumatoid disease. These are: (1) the classical form of Wegener's disease in which granulomatosis in the lung is associated with similar lesions in the upper respiratory tract and elsewhere together with glomerulonephritis (Wegener, 1936, 1939); (2) Wegener-type lesions limited to the lung (Carrington and Liebow, 1966); (3) lymphomatoid granulomatosis (Liebow, Carrington, and Friedman, 1972); (4) necrotizing 'sarcoid' angiitis and granulomatosis (NSG), and (5) bronchocentric granulomatosis (Katzenstein, Liebow, and Friedman, 1975). While the first three types are well documented, NSG and bronchocentric granulomatosis possibly represent previously unrecognized or even new entities. Certainly most practitioners will be unfamiliar with these diseases. This report describes a case of NSG which presented recently as a peripheral lung mass initially thought to be a neoplasm.

\section{CASE REPORT}

A 63-year-old underground station master presented in March 1974 with an 18-month his:ory of episodic tightness in the chest, mainly on exertion. He had a morning cough with whitish sputum but was dyspnoeic only on severe exertion. He was otherwise well and had not lost weight.
There was no history of allergy or exposure t\$ industrial pulmonary hazards. He smoked 20 cigarettes per day. A urinary tract infection ha 9 been treated in 1967, at which time chest radiog graphy and pyelography were normal. In 1971 he developed erythematous skin lesions on the legs and biopsy of these showed atrophy of the dermis but no arteritis, necrosis, inflammation or sca设 ring. These lesions subsequently resolved wit application of a steroid cream.

On examination he was a fit-looking slight $\hat{\varphi}$ obese man with no cyanosis or clubbing. No en larged lymph nodes were palpable. The bloog pressure was $135 / 75 \mathrm{mmHg}$ and the pulse and heart sounds were normal. Widespread wheezes. were present over both lungs. There were no abnormal signs in the abdomen. Investigations showed: Hb $16.4 \mathrm{~g} / \mathrm{dl}$; WBC $8600 / \mathrm{mm}^{3}$ witit $67 \%$ neutrophils, $26 \%$ lymphocytes, $5 \%$ mona cytes, and $2 \%$ eosinophils; ESR $8 \mathrm{~mm} / \mathrm{h}$; platele normal. Serum sodium was $134 \mathrm{mmol} / 1$, potassium $3.7 \mathrm{mmol} / 1$, bicarbonate $21 \mathrm{mmol} / 1$, and bloog urea $6.8 \mathrm{mmol} / \mathrm{l}$.

Chest radiography (Fig. 1) showed an ill-define mass in the posterior segment of the right uppot lobe, the appearances of which were consideref to be those of a neoplasm. With this presumptive diagnosis, a right thoracotomy was performed 画 April 1974. There were no adhesions but the visceral pleura was thickened and there was 


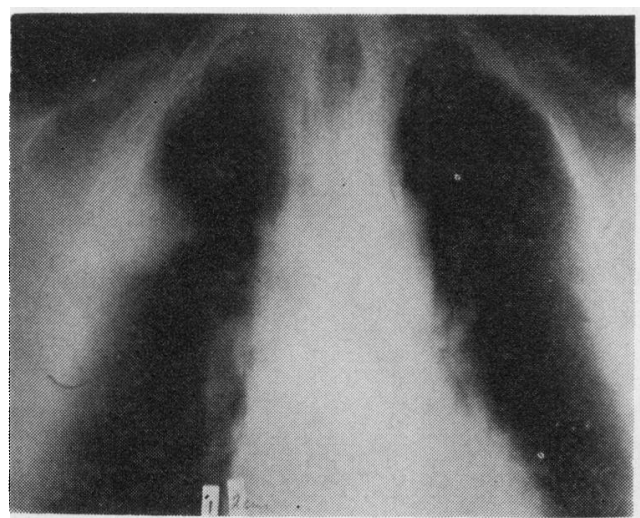

FIG. 1. Chest tomogram showing a poorly defined opacity in the right lateral lung field.

mass, approximately $5 \mathrm{~cm}$ in diameter, in the posierior segment of the right upper lobe close to the posterior end of the horizontal fissure. The oblique fissure was free except at its posterior end where the apical segment of the lower lobe was fused to the posterior segment of the upper lobe. A right upper and middle lobectomy was performed.

Examination of the excised specimen showed that over the lateral surface of the upper lobe there was a densely fibrotic scar which extended into the lung to a depth of $2 \mathrm{~cm}$ at its centre point. It was wedge-shaped with its base abutting on the pleural surface. About this scar there was a cuff of pale tissue, $2 \mathrm{~cm}$ thick, with an irregular outline (Fig. 2) but the main bronchi showed no evidence of tumour and elsewhere the lung appeared normal. The hilar lymph nodes measured up to $1 \mathrm{~cm}$ in diameter.

A preliminary histological examination showed that the lung tissue about the scar contained numerous epithelioid and giant-cell granulomata (Fig. 3). No caseation was seen, but it was thought that tuberculosis was the most likely of the many granulomatous processes which might have produced such changes. As the patient had then developed a severe postoperative pneumonia he was treated with antituberculosis chemotherapy. However, he failed to improve and when sputum culture demonstrated Haemophilus influenzae, ampicillin was added to the chemotherapy. Sputum and urine specimens showed no evidence of tubercle bacilli on microscopy or subsequent culture, and no mycobacteria nor fungi were found in specially stained sections of the resected tissue.

Histological examination of further areas

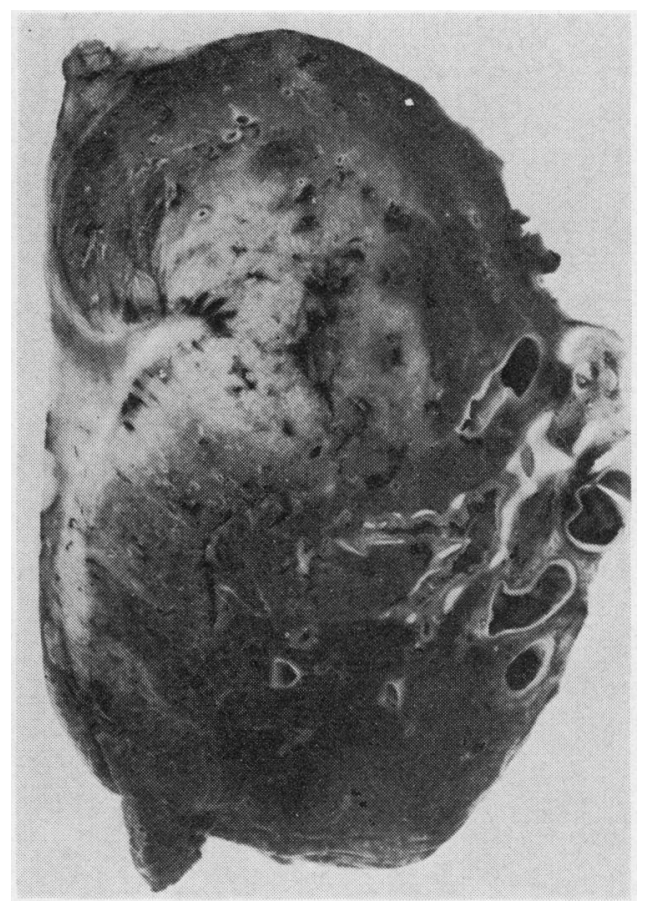

FIG. 2. Part of the resected specimen showing a central wedge-shaped scar surrounded by an ill-defined zone of pale inflammatory tissue.

showed abundant granulation tissue densely infiltrated by lymphocytes and plasma cells in addition to the many tuberculoid granulomas previously noted, and although the granulomas were non-caseating there were a few foci of necrosis in the non-specific inflammatory granulation tissue. These were walled off by densely hyaline fibrous tissue (Fig. 4) and may represent an older pre-existent lesion. A feature overlooked on preliminary examination was involvement of blood vessels by the granulomatous process. Many medium-sized arteries and veins showed infiltration of their walls by lymphocytes and epithelioid and Langhans-type giant cells, sometimes forming focal granulomas (Fig. 5), but often distributed more loosely within the vessel wall. All coats were involved and there was fibrous thickening of the intima with narrowing or obliteration of the lumen. The lung tissue elsewhere appeared normal, and the hilar lymph nodes showed sinus histiocytosis only. No tumour was found. The microscopic appearances were thought to correspond to those of NSG, and this was later confirmed by Dr. A. A. Liebow. Antituberculosis therapy was therefore withdrawn. The 


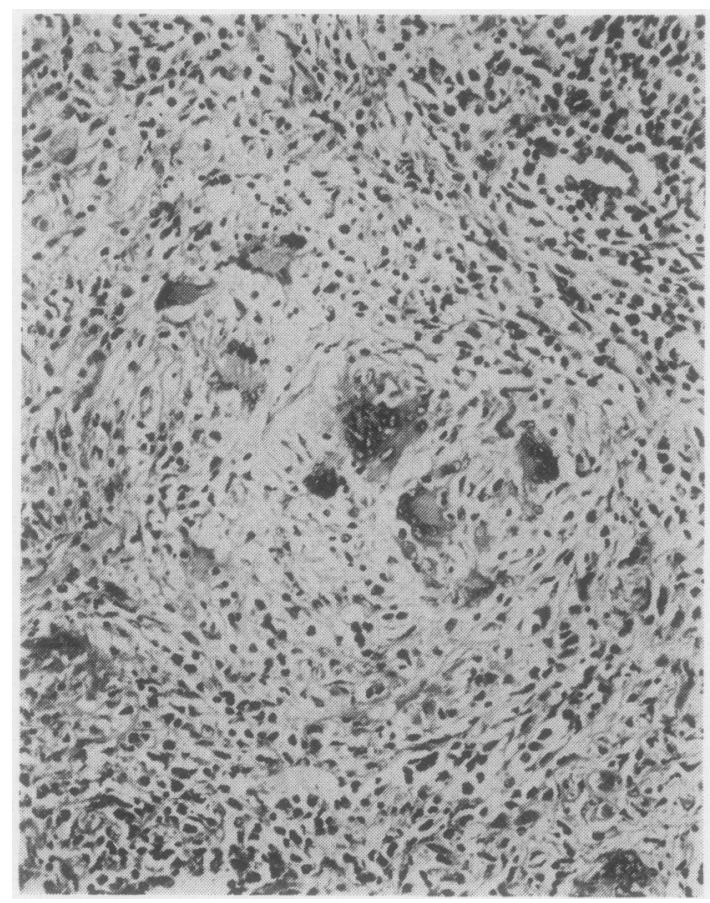

FIG. 3. One of the many non-caseating 'sarcoidal' giant-cell granulomas. Haematoxylin and eosin $(H$ and $E \times 160$ ).

patient made a steady recovery and was well and free from pain at follow-up two months later.

Six months after lobectomy the patient was again admitted to hospital with a three-day history of increasing shortness of breath. On examination he was gravely ill, dyspnoeic at rest, and centrally cyanosed, and coarse crepitations were heard throughout both lungs. An arterial blood gas determination showed a respiratory acidosis with hypoxaemia and hypercapnia $\left(\mathrm{pH} 7 \cdot 23, \mathrm{Po}_{2} 62\right.$, $\mathrm{PCO}_{2} 55 \mathrm{mmHg}$ ). A chest radiograph showed bilateral bronchopneumonia, and treatment with ampicillin, $500 \mathrm{mg}$ six-hourly, and hydrocortisone, $200 \mathrm{mg}$ six-hourly, was started. Within 20 hours the patient's condition improved dramatically, even though intensive physiotherapy had failed to produce any sputum, and this suggested that he may have been suffering from a recrudescence of the granulomatosis rather than pneumonia.

A further radiograph showed considerable clearing of the chest but a mass was now evident at the left hilum. Biopsy of an enlarged cervical lymph node showed metastatic oat-cell carcinoma and it was assumed that the hilar mass was neoplastic. Both hilar regions, mediastinum, and

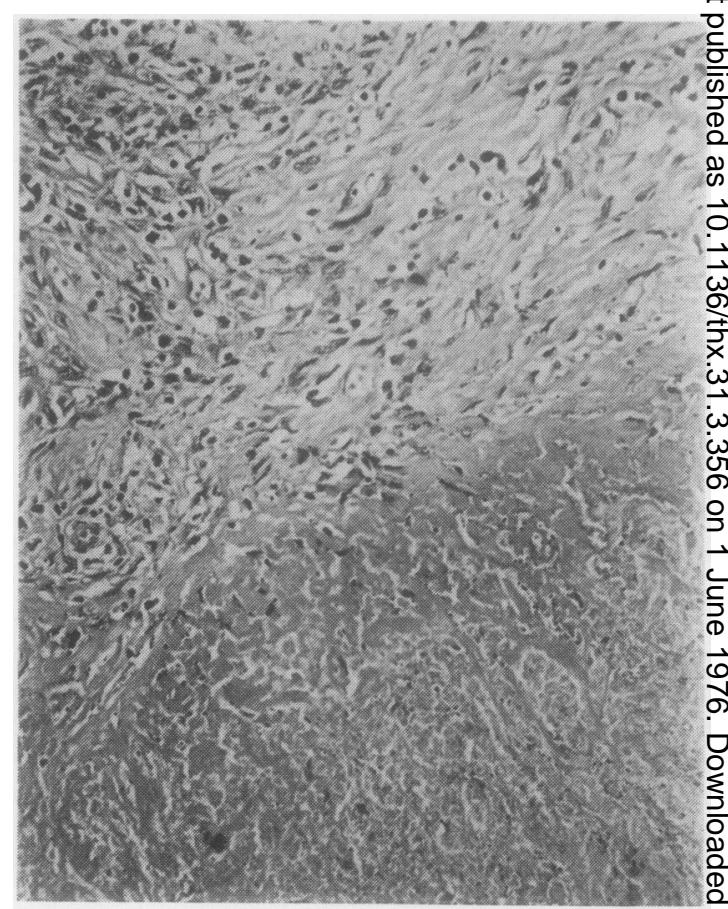

FIG. 4. A necrotic focus surrounded by hyalin $\overrightarrow{\mathrm{S}^{-}}$ fibrous tissue ( $H$ and $E \times 160$ ).

neck were irradiated (5400 rads over 25 days) following which his general condition remained satisfactory until two months after admissionx when he deteriorated and died. At necropsy there? were hepatic metastases but in the lungs tumour was limited to a small focus in the periphery of the left upper lobe. Elsewhere the lungs showed diffuse interstitial fibrosis with hyaline membranes? and a prominent alveolar epithelium compatible? with radiation damage. Massive fibrosis with obliteration of the alveolar architecture, as found N in NSG, was not evident, and the appearances provided no support for a recrudescence of this disease.

\section{DISCUSSION}

Our 63-year-old man may be compared with the 11 cases of NSG previously described by Liebow. (1973) who were young or middle-aged, the oldest being 59 years, and of equal sex distribution. Two of Liebow's patients, both with radiographically localized unilateral disease, were asymptomatic, $\stackrel{\mathbb{Q}}{ }$ but most had symptoms such as cough, fever, sweats, malaise, dyspnoea, and pleuritic pain sug- $\Omega$ gesting infection. The physical findings weree 


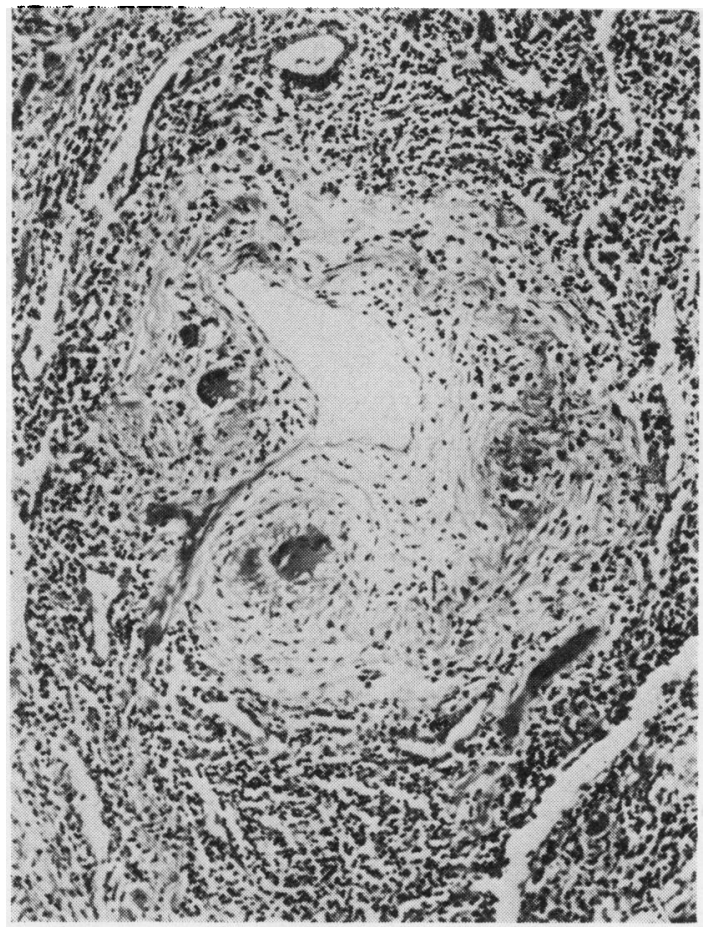

FIG. 5. An artery showing discrete giant-cell granulomas within its thickened walls $(H$ and $E \times 125)$.

minimal, and there was no evidence of generalized disease. Chest radiographs generally showed multiple nodules or ill-defined infiltrates but the two asymptomatic patients had localized disease, and our case would appear to correspond to these, the cough, wheezes, and sputum production being attributable to chronic bronchitis.

Follow-up information available at present suggests that the course of NSG is relatively benign and that the disease is possibly responsive to steroids. However, it is clear that when the process is localized the radiological appearances may lead to a presumptive diagnosis of carcinoma and subsequent lung resection. Preliminary percutaneous biopsy of all localized peripheral lung 'tumours' would obviate some unnecessary lung resections. The histological characteristics are such that NSG may be confused with other granulomatous conditions, in particular sarcoidosis and tuberculosis. Familiarity with the condition will lead pathologists to pay particular attention to the blood vessels, for, together with the sarcoidal granulomas, angiitis is the hallmark of NSG. The definite diagnosis will depend on histological examination of an adequate biopsy specimen and this may require thoracotomy. In view of the uncertain aetiology culture of the operative specimen is also desirable.

The aetiology of NSG is obscure and the possibility of a relationship to Boeck's sarcoidosis arises. Minute granulomas may be found in the hilar lymph nodes in NSG but there is no significant lymph node enlargement and extra pulmonary sites are not otherwise involved. In pulmonary sarcoidosis the blood vessels are not usually affected, although vascular involvement was prominent in the cases described by Bottcher (1959) and Michaels, Brown, and Cory-Wright (1960). In the first case described by Michaels and his colleagues the process was confined to the lung, where it was associated with bronchiectasis. This patient possibly represents another case of NSG. These authors speculate that the bronchiectasis may have played an aetiological role in the development of the 'sarcoidosis', but in view of Liebow's description of airway involvement in NSG with consequent obstructive pneumonitis a reverse relationship is equally possible. Other diseases which share morphological features with NSG include the limiled variant of Wegener's granulomatosis distinguished by its paucity of sarcoid lesions, and giant-cell arteritis distinguished by its anatomical distribution and lack of surrounding chronic inflammatory granulation tissue.

Boeck's sarcoid, Wegener's granulomatosis, and giant-cell arteritis are widely differing diseases in which, though little is known of their aetiology, indeterminate immune disturbances are widely favoured. In NSG the presence of an angiitis together with epithelioid and giant-cell granulomata is also very suggestive of hypersensitivity phenomena. A possibly immune process concentrated on the lung suggests that an inhaled antigen may have been the initiating factor but so far at least in NSG none has been identified. The association in our case with an oat-cell carcinoma of the opposite lung is probably coincidental but tumour antigens could conceivably have initiated the granulomatosis, just as they are suspected of causing renal glomerular damage (Higgins, Randall, and Still, 1974).

We are indebted to Professors A. A. Liebow and $\mathrm{H}$. Spencer for advice on the histological diagnosis.

\section{REFERENCES}

Bottcher, E. (1959). Disseminated sarcoidosis with a marked granulomatous arteritis. Archives of Pathology, 68, 419. 
Carrington, C. B. and Liebow, A. A. (1966). Limited forms of angiitis and granulomatosis of Wegener's type. American Journal of Medicine, 41, 497.

Higgins, M. R., Randall, R. E., Jr., and Still, W. J. S. (1974). Nephrotic syndrome with oat-cell carcinoma. British Medical Journal, 3, 450.

Katzenstein, A. L., Liebow, A. A., and Friedman, P. J. (1975). Bronchocentric granulomatosis, mucoid impaction and hypersensitivity reactions to fungi. American Review of Respiratory Disease, 111, 497.

Liebow, A. A. (1973). Pulmonary angiitis and granulomatosis. American Review of Respiratory Disease, 108, 1.

Carrington, C. B., and Friedman, P. J. (1972). Lymphomatoid granulomatosis. Human Pathology, 3, 457.
Michaels, L., Brown, N. J., and Cory-Wright, (1960). Arterial changes in pulmonary sarcoidosis Archives of Pathology, 69, 741.

Wegener, F. (1936). Über generalisierte septisch Gefasserkrankungen. Verhandlungen der Deü schen Gesellschaft für Pathologie, 29, 202.

(1939). Uber eine eigenartige rhinogeneGranulomatose mit besonderer Beteiligung des Arteriensystems und der Nieren. Beiträge zur pathologischen Anatomie und zur allgemeine Pathologie, 102, 36.

Requests for reprints to: Dr. B. Corrin, Departmento of Morbid Anatomy, St. Thomas' Hospital Medic你 School, London SE1 7EH. 